\title{
Gene activation of CEBPA using saRNA: preclinical studies of the first in human saRNA drug candidate for liver cancer
}

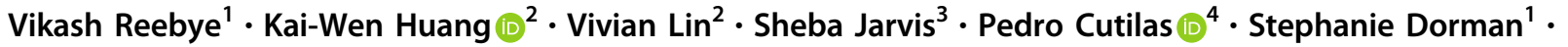 \\ Simona Ciriello ${ }^{5}$ Pinelopi Andrikakou ${ }^{1} \cdot$ Jon Voutila $^{5} \cdot$ Pal Saetrom $^{6,7} \cdot$ Paul J. Mintz $^{1} \cdot$ Isabella Reccia ${ }^{1}$. \\ John J. Rossi ${ }^{8} \cdot$ Hans Huber $^{9} \cdot$ Robert Habib $^{5} \cdot$ Nikos Kostomitsopoulos $^{10} \cdot$ David C. Blakey $^{5}$. Nagy A. Habib ${ }^{1}$
}

Received: 2 July 2017 / Revised: 2 December 2017 / Accepted: 12 December 2017 / Published online: 7 March 2018

(c) The Author(s) 2018. This article is published with open access

\begin{abstract}
Liver diseases are a growing epidemic worldwide. If unresolved, liver fibrosis develops and can lead to cirrhosis and clinical decompensation. Around 5\% of cirrhotic liver diseased patients develop hepatocellular carcinoma (HCC), which in its advanced stages has limited therapeutic options and negative survival outcomes. CEPBA is a master regulator of hepatic function where its expression is known to be suppressed in many forms of liver disease including HCC. Injection of MTL-CEBPA, a small activating RNA oligonucleotide therapy (CEBPA-51) formulated in liposomal nanoparticles (NOV340- SMARTICLES) upregulates hepatic CEBPA expression. Here we show how MTL-CEBPA therapy promotes disease reversal in rodent models of cirrhosis, fibrosis, hepatosteatosis, and significantly reduces tumor burden in cirrhotic HCC. Restoration of liver function markers were observed in a carbon-tetrachloride-induced rat model of fibrosis following 2 weeks of MTL-CEBPA therapy. At 14 weeks, animals showed reduction in ascites and enhanced survival rates. MTLCEBPA reversed changes associated with hepatosteatosis in non-alcoholic methionine and cholic-deficient diet-induced steaotic liver disease. In diethylnitrosamine induced cirrhotic HCC rats, MTL-CEBPA treatment led to a significant reduction in tumor burden. The data included here and the rapid adoption of MTL-CEBPA into a Phase 1 study may lead to new therapeutic oligonucleotides for undruggable diseases.
\end{abstract}

Electronic supplementary material The online version of this article (https://doi.org/10.1038/s41388-018-0126-2) contains supplementary material, which is available to authorized users.

\footnotetext{
$\triangle$ Vikash Reebye

v.reebye@ic.ac.uk

Nagy A. Habib

nagy.habib@ic.ac.uk

1 Department of Surgery, Imperial College London, London, UK

2 Department of Surgery and Hepatitis Research and Graduate Institute of Clinical Medicine, National Taiwan University, Taipei, Taiwan

3 Department of Surgery and Cancer, Imperial College London, London, UK

4 Cell Signalling and Proteomics Group, Centre for HaematoOncology, Barts Cancer Institute, Queen Mary University of London, London, UK
}

\section{Introduction}

Liver disease is caused by a multitude of components including obesity, alcoholism, and viral hepatitis. These synergistically culminate in an asymptomatic "compensated cirrhosis" stage where the average life expectancy of patients is $10-13$ years. This is usually followed by a

5 MiNA Therapeutics Ltd, London, UK

6 Department of Cancer Research and Molecular Medicine, Norwegian University of Science and Technology, Trondheim, Norway

7 Department of Computer and Information Science, Norwegian University of Science and Technology, Trondheim, Norway

8 Department of Molecular and Cellular Biology, Beckman Research Institute, City of Hope, Duarte, CA, USA

9 BioTD Strategies, LLC, Lansdale, PA, USA

10 Biomedical Research Foundation of the Academy of Athens, Centre of Clinical, Experimental Surgery and Translational Research, Athens, Greece 
rapidly progressive phase of liver dysfunction termed "decompensated cirrhosis" where the average life expectancy is reduced to as low as 2 years [1].

In the United States, there were 658,000 hospitalization associated with cirrhosis in 2011, which culminated in $\$ 9.8$ billion dollars in cost [2]. Despite recent medical advances, liver cirrhosis remains a poorly treated stage of liver disease and in 2013 claimed 1.2 million lives globally. Liver cirrhosis is now the 8th leading cause of death in the United States and the 13th leading cause of death globally, with a $46 \%$ increase in worldwide mortality over the past two decades [1].

Progression of cirrhosis can be accelerated by the development of other complications which include renal impairment, sepsis, increasing viral titre and development of hepatocellular carcinoma (HCC) [3]. According to longterm outcome studies, the severity of liver fibrosis is the most important predictor for patient outcome [4].

Unresolved fibrosis ultimately leads to a decompensated liver where the only treatment at this stage is liver transplantation. However, the scarcity of organ donors, coupled with the increasing incidence of chronic liver disease which is intractable to conventional therapeutic compounds, poses a major threat for patient survival. Patients who progress to HCC end up with a severely compromised liver owing to the natural progression of the disease, which decreases their opportunity to meet eligibility for curative surgery, transarterial chemoembolization or sorafenib treatment.

An improvement of liver function would be expected to benefit the general condition and Child Pugh status of patients; their quality of life and more significantly allow them to re-enter eligibility criteria for surgical interventions, which would ultimately enhance chances of survival.

A treatment that reverses fibrosis, improves liver function, whilst inhibiting tumor growth would be a major therapeutic breakthrough and here we present data for a novel RNA-based drug candidate in the form of small activating RNAs (saRNAs), which is currently in a phase I clinical trial for patients with advanced cirrhotic HCC (ClinicalTrials.gov-NCT0271601).

saRNAs differ from other RNA-based therapeutics in that they upregulate, rather than inhibit the expression of their therapeutic target [5]. The mechanism of action involves loading of saRNA into the Argonaute 2 (Ago2) protein complex, translocation into the nucleus, binding to the genomic target locus and promoting increased transcriptional activity by RNA Polymerase $2[6,13]$.

Here we report an saRNA therapy that was designed to increase production of CEBPA (CCAAT/enhancer-binding protein alpha), a master transcriptional factor in the liver, which resets the natural gene regulatory mechanism of hepatocytes to reduce fibrosis and reverse liver dysfunction.
The potential benefits of increasing expression levels of C/EBP- $\alpha$ have been considered in the literature to date. Downregulation of CEBPA is well known across many different liver disease models including fibrosis and cirrhosis [7, 8]. Furthermore the importance of CEBPA activity in normal liver function is observed in "old age"-related liver disease models, where hyperphosphorylation of CEBPA at serine residue 193 diminished transcriptional activity [9]. In transgenic CEBPA knock-out models, animals appear to develop liver tumors at an increased rate $[10,11]$.

In order to validate the therapeutic benefit of increasing CEBPA expression in liver disease, we developed a therapeutic oligonucleotide (CEBPA-saRNA) for intravenous delivery using nanoparticles with high tropsim to the liver [12]. The successful outcome of this study led us to further develop CEBPA-saRNA into a GMP grade manufactured clinical candidate (CEBPA-51) with early indications to show high target specificity and reliance on $\mathrm{Ago} 2$ and components of the RNA-interacting transcriptional activating complex [13]. This therapeutically viable saRNA has been encased in liposomal nanoparticles (SMARTICLES) [14] for the first in-human trial to study the outcome of targeting CEBPA activation using saRNAs in patients with HCC (clinicaltrials.gov-NCT0271601).

Here, we report the preclinical data obtained from treating multiple liver disease models with the new therapeutic candidate MTL-CEBPA. We show how restoration of CEBPA expression in the liver markedly reverses disease in fibrosis and steatosis models, whilst improving liver function tests and ultimately increasing survival rates in long-term fibrosis $\left(\mathrm{CCl}_{4^{-}}\right.$induced rats). The data included here and the rapid adoption of MTL-CEBPA into a Phase 1 study may lead to new therapeutic oligonucleotides for undruggable diseases.

\section{Results}

\section{In vitro activation of C/EBP-a by CEBPA-51}

The ability of CEBPA-51 to increase CEBPA mRNA expression in vitro was demonstrated in murine (AML12); rat (Clone 9) liver cell line, human (HEPG2) liver cells, and primary human hepatocytes, (Fig. 1a). In each case, transfection of the newly GMP developed CEBPA-51 saRNA significantly increased CEBPA mRNA levels by about twofold, whereas the non-specific control RNA oligonucleotide (siFLUC) was inactive. In human primary hepatocytes, transfection of CEBPA-51 showed increased expression of CEBPA mRNA and significant changes to expression of downstream targets of CEBPA. These included key liver function factors including albumin, 

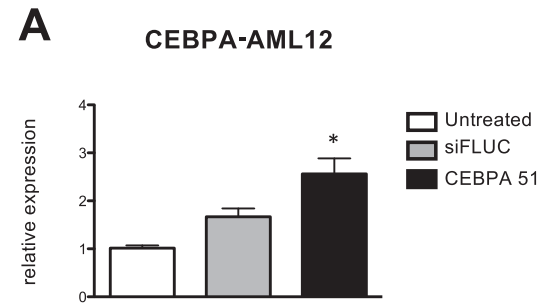

CEBPA- Primary human hepatocyte

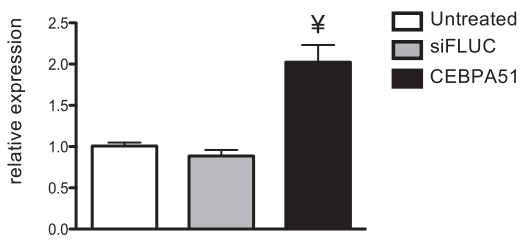

B

Albumin

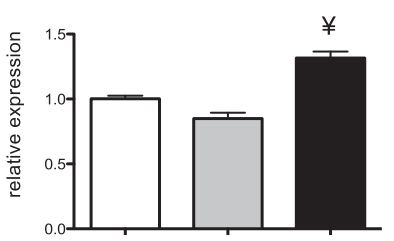

$\square$ Untreated

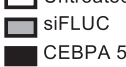

C

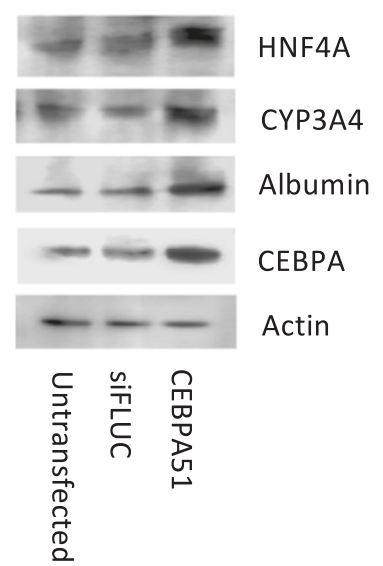

Fig. 1 Transcriptional activity of CEBPA-51 in vitro. a CEBPA-51 saRNA transfection increases CEBPA mRNA in murine AML12, Rattus (Clone 9), Human (HEPG2) and human (Primary hepatocytes). Key factors for liver function were screened in CEBPA-51 transfected primary human hepatocytes. These included b albumin, HNF4A (Hepatocyte nuclear factor 4-alpha), and CYP3A4 (Cytochrome P450 $3 \mathrm{~A} 4)$. Data are expressed as mean \pm SEM. $¥=p(<0.00027)$ and $*=p$

HNF4A, and CYP3A4 (Fig. 1b). Increased expression of these factors were also measured at the protein level by western blotting (Fig. 1c).

\section{In vivo delivery of MTL-CEBPA reduces tumor burden in DEN-induced cirrhotic HCC}

The newly GMP manufactured oligonucleotide formulated in SMARTICLES; MTL-CEBPA was tested in the same
(0.0033). c A western blot panel probed with antibody specific to HNF4A (Abcam; ab41898), CYP3A4 (Abcam; ab155029), Albumin (Abcam; ab106582), and CEBPA (CELL Signalling; 8178) showed visible increase in protein expression in cells transfected with CEBPA51. Actin expression (Abcam: ab8226) was used as protein loading control across each of the lanes

diethylnitrosamine (DEN)-induced cirrhotic HCC rat model as previously reported with CEBPA-saRNA [15]. This was compared to treatment with an unspecific oligonucleotide also formulated in SMARTICLES (NOV340/FLUC) (Fig. 2a). In brief, DEN was administered for 9 weeks in male Wistar rats followed by a 2 week wash-out period. At week 11 , animals were randomized for each experimental arm. Tumor-bearing animals were treated with three doses of either NOV340/FLUC control or MTL-CEBPA at days 1, 3, 

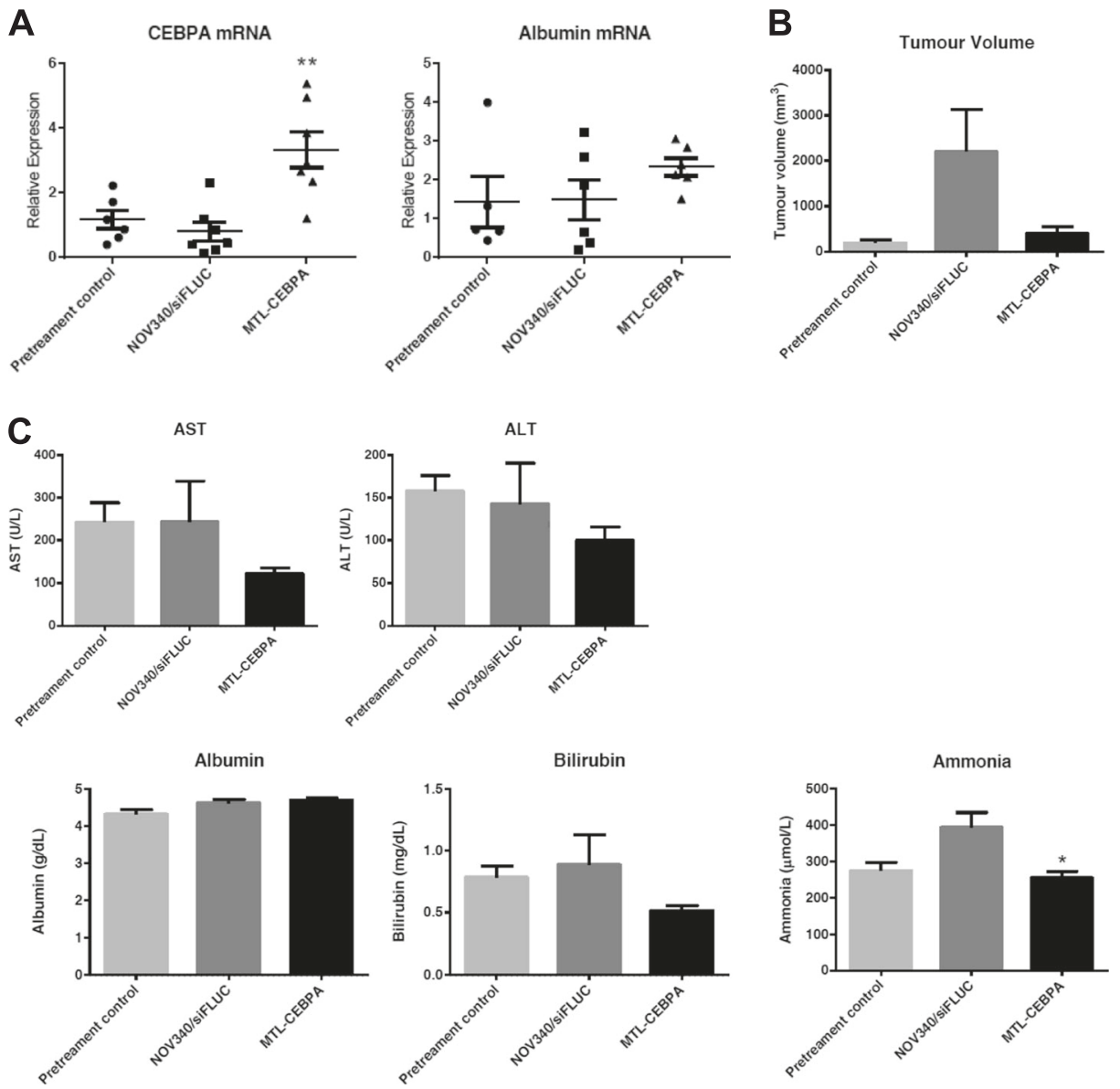

Fig. 2 MTL-CEBPA increases liver expression of CEBPA in DENinduced HCC Wistar rats. a MTL-CEBPA showed a significant increase in CEBPA mRNA: **, $p<0.001$. Albumin showed a tendency to increase after MTL-CEBPA treatment, however this was not statistically significant $(p=0.1769)$. Tumor nodule size and liver function tests were assessed in the DEN-induced cirrhotic HCC animals. b Liver tumor nodule size was measured from the biopsies across the pre-treatment control and two treatment groups. The average tumor size in the DEN only group (pre-treatment) was $193( \pm 63)$ $\mathrm{mm}^{3}$. Over the 12-day course of the study period, the tumor size of animals treated with NOV340/FLUC at time of termination increased to $2204( \pm 1031) \mathrm{mm}^{3}$. All animals treated with MTL-CEBPA showed a slower rate of increase with an average volume of $401( \pm 181) \mathrm{mm}^{3}$ ( $p=0.107$ vs. NOV340/FLUC control). $\mathbf{c}$ Liver function parameters were measured for AST, ALT, albumin, and bilirubin. All showed a $30-50 \%$ decrease over the short study period. Ammonia decreased significantly despite the small group size. $(*, p<0.02)$

parameters for AST, ALT, bilirubin, and ammonia levels all decreased between 30 and 50\% over this short course of MTL-CEBPA treatment. The measured ammonia decrease was statistically significant $(p<0.02)$ given the small group size and variability in tumor burden (Fig.2c).

\section{Activity of MTL-CEBPA (the CEBPA-51 clinical candidate) improves liver parameters in $\mathrm{CCl}_{4}$ model of liver failure}

Having recapitulated the same effects of CEBPA-saRNA as previously published, our new clinical candidate MTLCEBPA was then investigated across different models of liver diseases. MTL-CEBPA was injected into a liver failure rat model exposed to the hepatotoxin carbon-tetrachloride $\left(\mathrm{CCl}_{4}\right)$. Sprague Dawley rats were treated with $\mathrm{CCl}_{4}$ for 


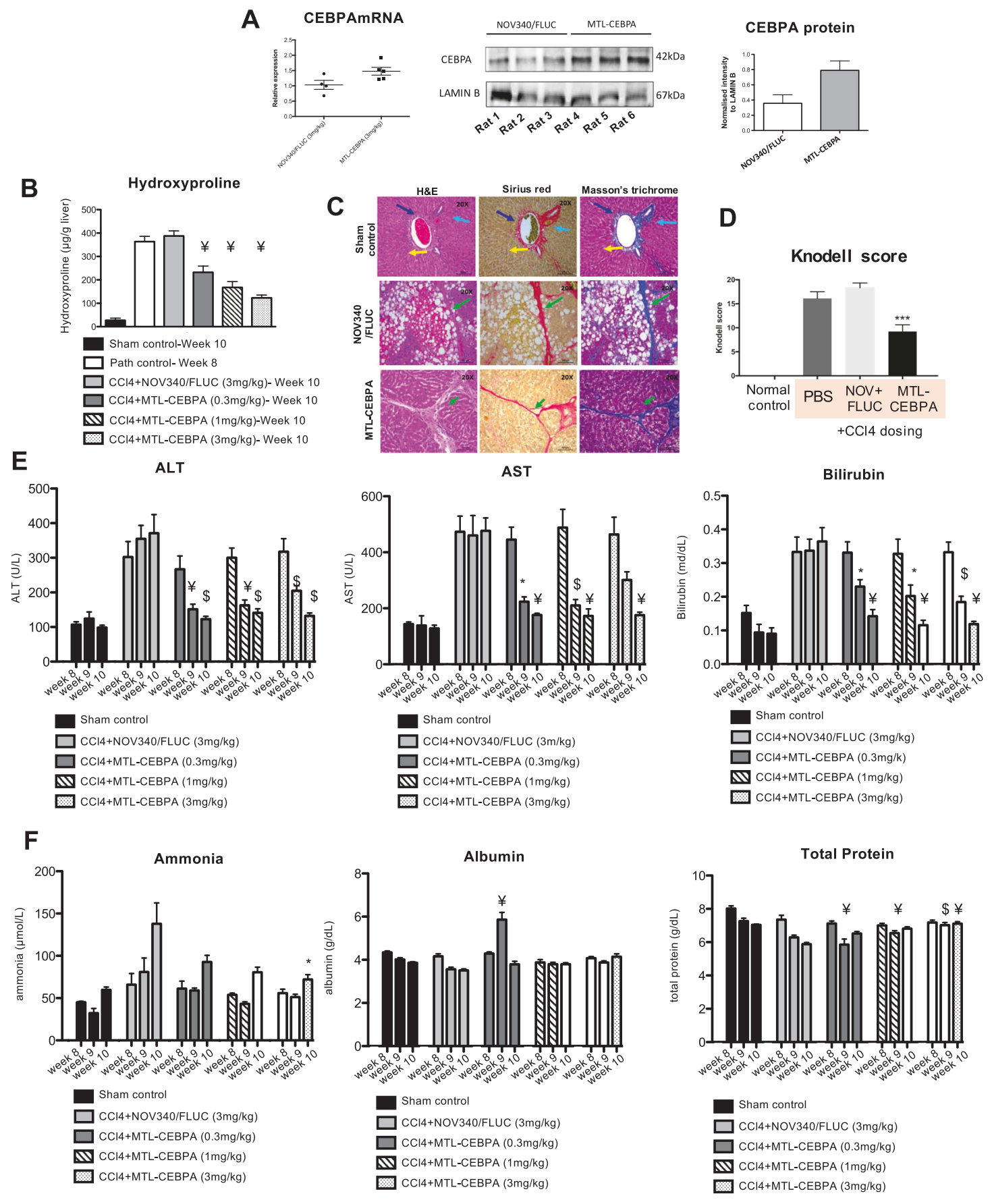

G
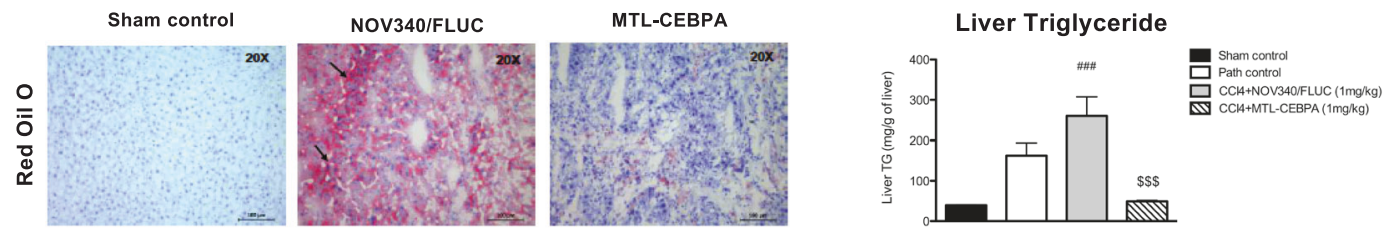
Fig. 3 The effect of MTL-CEBPA treatment in $\mathrm{CCl}_{4}$ induced liver damage. a MTL-CEBPA administration in rats caused an increase in liver CEBPA mRNA expression and protein. Total RNA and protein were extracted from the liver of treated animals. Protein levels were assessed using Image $\mathbf{J}$ to perform band densitometry measurements. b MTL-CEBPA caused a dose dependent decrease in hydroxyproline at week 10 when compared to NOV340/Fluc control. Normal hydroxyproline levels were observed in the sham control group at week 10 compared to the pathological ('Path control') group at week 8. Data is expressed as mean \pm SEM vs. NOV340/FLUC. $¥=p(<0.0002)$. c Liver sections at week 11 ('Path control') and week 13 ('Sham control') were stained with H\&E, Sirius red and Masson's trichrome. (Magnification- $-\times 20$, Sham control, NOV304/FLUC, and MTLCEBPA treatment groups). Sham control animals revealed normal architecture with hepatocytes arranged in the form of cords (light blue arrow) radiating away from the central vein (dark blue arrow). Normal distribution of sinusoidal spaces (yellow arrow) was observed. In the NOV340/FLUC treatment groups fibrous bands (green arrow) extended from one portal area to the adjacent or to the central vein. In the MTL-CEBPA treatment group, thinner fibrous bands (green arrow) were observed, when compared to NOV340/FLUC group. d The severity of hepatic necrosis was assessed. MTL-CEBPA treatment caused a significant reduction in Knodell score (activity index). ${ }^{* * *} p<0.001$ compared to NOV340/FLUC. Statistical analysis: oneway ANOVA followed by Tukey's multiple comparison test. The effect of MTL-CEBPA on liver function tests were assessed. e ALT, AST, and bilirubin were measured and showed improvement at all doses of MTL-CEBPA. f Ammonia levels reversed despite continued exposure to $\mathrm{CCl}_{4}$. Although minor changes in levels of albumin and total protein (globulin) were observed; this appeared to be stabilized over the course of treatment. Data are expressed as mean \pm SEM vs. NOV340/FLUC. $*=p(0.0033) ; ¥=p(<0.00027) ; \quad \mathrm{S}=p(0.0006)$. The effect of MTL-CEBPA on fat deposit in the liver. $g$ Cryosections of the liver were processed for Oil red O staining. Sham control animals showed absence of positively stained Oil red O-positive fat globules. In the NOV340/FLUC group, there was an abundant number of Oil red O-positive fat globules. This decreased in the MTL-CEBPA treated groups. Liver triglyceride levels were measured at week 13 and showed a significant reduction in the MTL-CEBPA treated group. $\#$ \#\#\# $p .01$ and ${ }^{\$ \$} p<0.001$ compared with NOV340/siFLUC. Oneway ANOVA followed by Tukey's multiple comparisons test

8 weeks and then randomized into four treatment groups ( $n=9$ in each group). This comprised of MTL-CEBPA at three doses of $0.3 \mathrm{mg} / \mathrm{kg} ; 1.0 \mathrm{mg} / \mathrm{kg}$, and $3.0 \mathrm{mg} / \mathrm{kg}$ bi-weekly for 2 weeks co-administered alongside constant exposure to $\mathrm{CCl}_{4}$. As control treatment, NOV340/FLUC was used at $3.0 \mathrm{mg} / \mathrm{kg}$. To ascertain disease progression, a $\mathrm{CCL}_{4}$-only exposed group was maintained as ('Path control') and compared with normal animals ('Sham control')-treated with olive oil.

Treatment with MTL-CEBPA showed increased liver expression of CEBPA mRNA and protein levels (Fig. 3a). Increased expression of CEBPA culminated in a significant reduction in fibrosis as measured by hydroxyproline levels, when compared to the pathological control group ('Path control'), whereas the NOV340/FLUC control had no impact (Fig. 3b). The reduction in hydroxyproline following MTL-CEBPA treatment was dose dependent. Liver tissues from animals in the MTL-CEBPA groups showed decreased histological fibrotic lesions when compared to the control group as shown here at $1.0 \mathrm{mg} / \mathrm{kg}$ of MTL-CEBPA (Fig. 3c, d).

Liver function tests demonstrated that MTL-CEBPA treatment reversed several disease parameters including ALT, AST, and bilirubin despite continued exposure to $\mathrm{CCl}_{4}$ (Fig. 3e). Ammonia levels significantly reduced after 2 weeks of treatment with MTL-CEBPA at all dose levels after just 1 week (Fig. 3f). Albumin, and total protein changes were minor in this model but appeared to be stabilized by MTL-CEBPA treatment (Fig. 3f). When examining liver pathology at the end of the study, it became apparent that MTL-CEBPA markedly reduced lipid accumulation as observed by the significant decrease in red oil-O staining and liver triglycerides shown here at $1.0 \mathrm{mg} / \mathrm{kg}$ of MTL-CEBPA treatment (Fig. $3 \mathrm{~g}$ ). Based on this initial study, the next strategy was to investigate the survival impact of MTL-CEBPA treatment in advanced cirrhotic animals where saRNA therapy would be extended to 14 weeks of bi-weekly injections.

\section{Treatment of MTL-CEBPA in advanced stages of liver disease prolongs survival and improves liver function parameters}

The long-term benefit of MTL-CEBPA on liver function and survival was assessed in animals chronically exposed to $\mathrm{CCl}_{4}$ for 36 weeks. Animals were divided into two groups ( $n=9$ in each group) comprising of a $\mathrm{CCl}_{4}$ treatment control only and a $\mathrm{CCl}_{4}+$ MTL-CEBPA treatment group. Oligonucleotide treatment commenced after animals were exposed to $\mathrm{CCl}_{4}$ for 8 weeks where MTL-CEBPA was maintained at bi-weekly injections for 14 weeks. After the final injection of MTL-CEBPA, animals were continually exposed to $\mathrm{CCl}_{4}$ for a further 14 weeks until cessation of the study.

Upon treatment withdrawal, at week 22, fewer than half of the animals in the untreated control group remained alive. There was a significant $78 \%$ increased survival benefit $(p<$ 0.001 - log rank test) in the MTL-CEBPA- treated group (Fig. 4a). Although all animals developed ascites; those in the control group rapidly escalated to grade 3 (visibly marked distension of the abdomen); all animals in the MTLCEBPA treatment group displayed ascites scores that were limited to Grade $1 / 2$ (with only mild to moderate symmetrical distension of the abdomen) (Fig. 4b).

Albumin and total protein levels remained elevated in the MTL-CEBPA-treated groups, when compared to untreated control (Fig. 4c, d). All animals treated with MTL-CEBPA also demonstrated reduction in liver damage markers including ALT, AST, and ammonia (Fig. 4e-g). MTLCEBPA-treated animals also displayed an improvement in obstructive liver function as indicated by reduced 
A

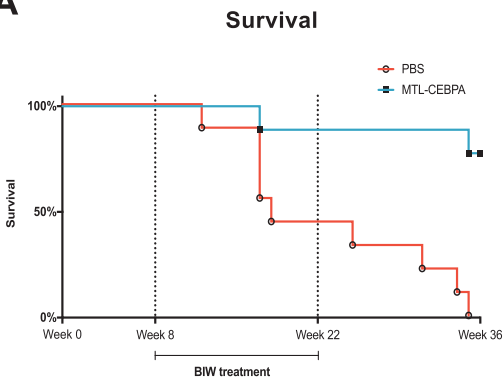

D

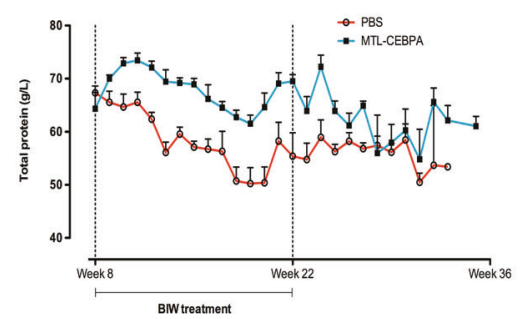

G

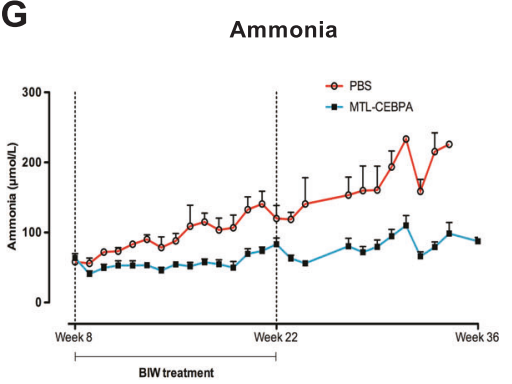

$\mathbf{J}$

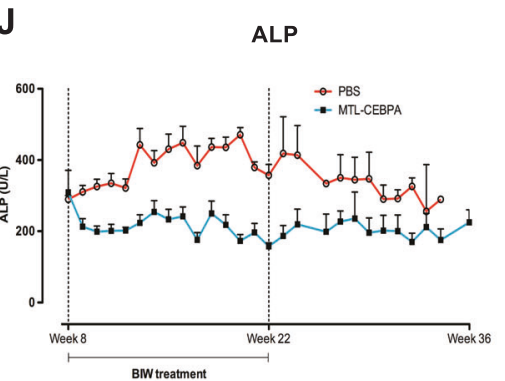

B

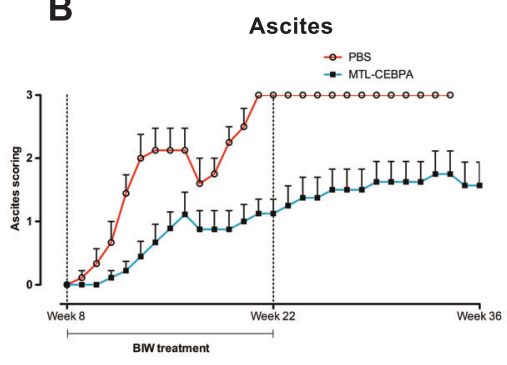

E

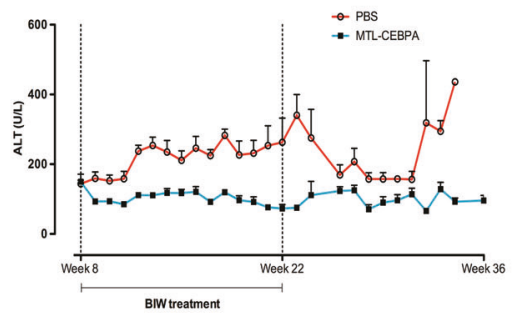

H

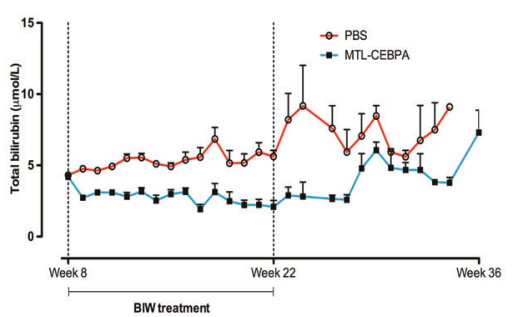

K

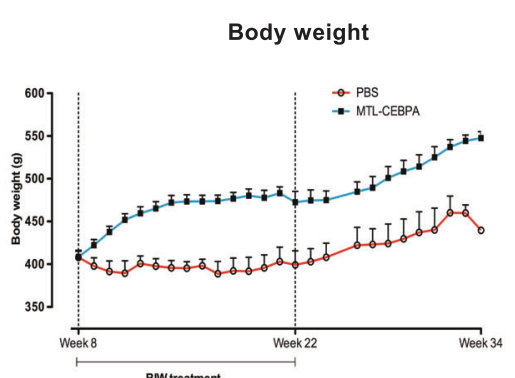

C

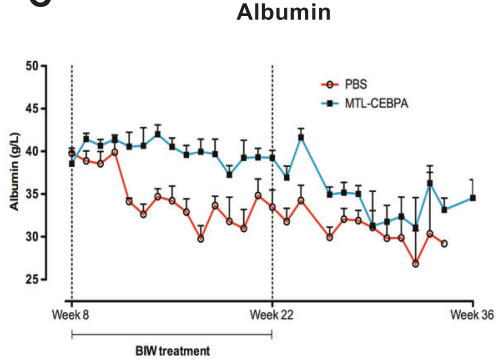

$\mathbf{F}$

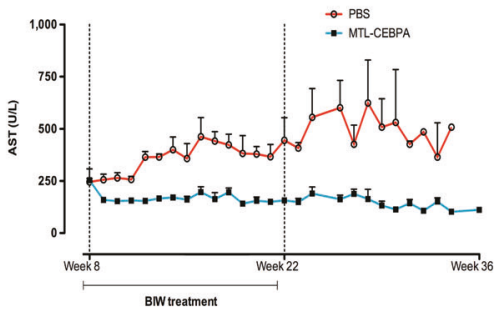

I

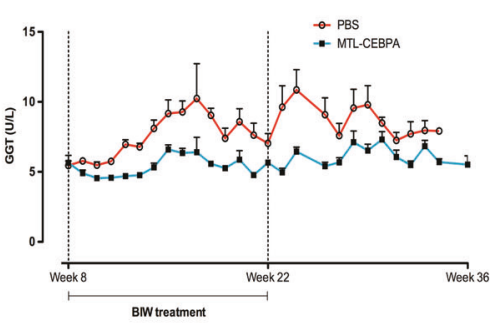

$\mathbf{L}$

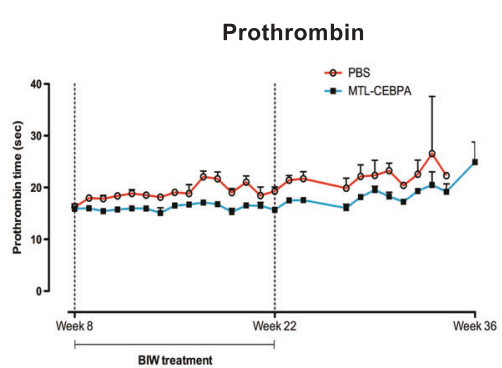

Fig. 4 The long-term effects of MTL-CEBPA on SD rats exposed to $\mathrm{CCl}_{4}$ for 36 weeks. a MTL-CEBPA treatment for 14 weeks caused a significant benefit to survival. b All animals had developed ascites which escalated to grade 3 in the PBS control group whereas in the MTL-CEBPA group this was limited to grade 1-2. (Ascites scoring = 1: Mild ascites only detected by palpation; 2 : moderate ascites causing moderate symmetrical distension of the abdomen and 3: large ascites cause visibly extended abdominal distension). Liver function tests on blood collected from SD rats exposed to long-term $\mathrm{CCL}_{4}$ induced toxicity. c Albumin and $\mathbf{d}$ total protein (globulin) remained raised when compared to the untreated control group. e ALT, f AST, g ammonia, $\mathbf{h}$ total bilirubin, $\mathbf{i}$ GGT and $\mathbf{j}$ ALP, $\mathbf{k}$ Body weight, and $\mathbf{l}$ prothrombin clotting time all collectively showed improvement in MTL-CEBPA treatment animals. All values represent mean \pm SEM

against $\mathrm{CCl}_{4}$-induced liver toxicity lasted for at least 6-8 weeks following withdrawal of treatment.

\section{Activity of MTL-CEBPA in a MCD diet model of NASH}

Having observed significant reduction in liver triglyceride levels from the $\mathrm{CCl}_{4}$ liver failure model and when (in vitro) 
A
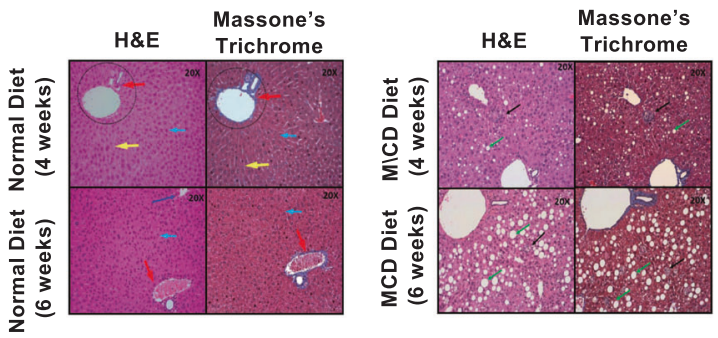

B
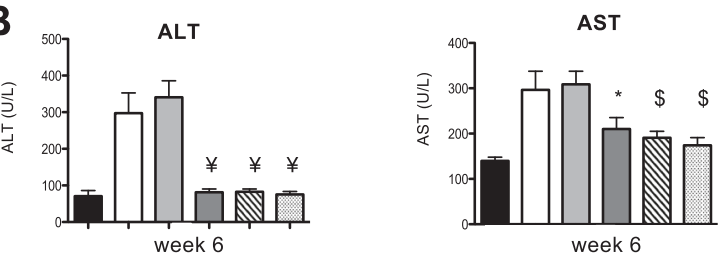

Normal Diet

$\square$ MCD Diet

$\square \mathrm{MCD}+\mathrm{NOV} 340 / \mathrm{FLUC}(3 \mathrm{mg} / \mathrm{kg})$

$\square$ MCD+MTL-CEBPA $(0.3 \mathrm{mg} / \mathrm{kg})$

MCD+MTL-CEBPA $(1 \mathrm{mg} / \mathrm{kg})$

MCD $+M T L-C E B P A(3 \mathrm{mg} / \mathrm{kg})$
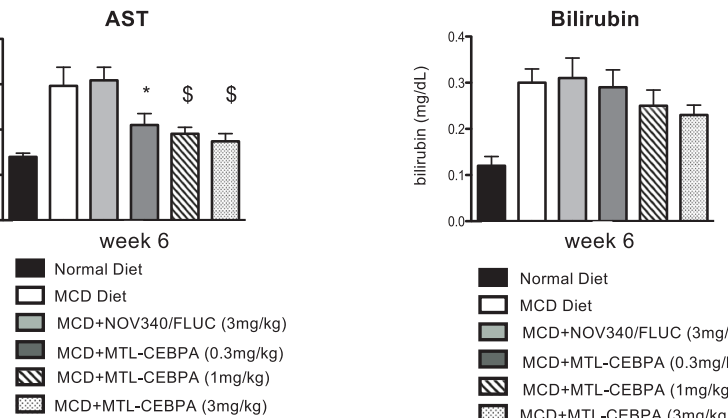

Normal Diet

$\square$ MCD Diet

$\square$ MCD+NOV340/FLUC $(3 \mathrm{mg} / \mathrm{kg})$

$\square$ MCD+MTL-CEBPA $(0.3 \mathrm{mg} / \mathrm{kg})$

М MCD+MTL-CEBPA $(1 \mathrm{mg} / \mathrm{kg})$

MCD + MTL-CEBPA $(3 \mathrm{mg} / \mathrm{kg})$
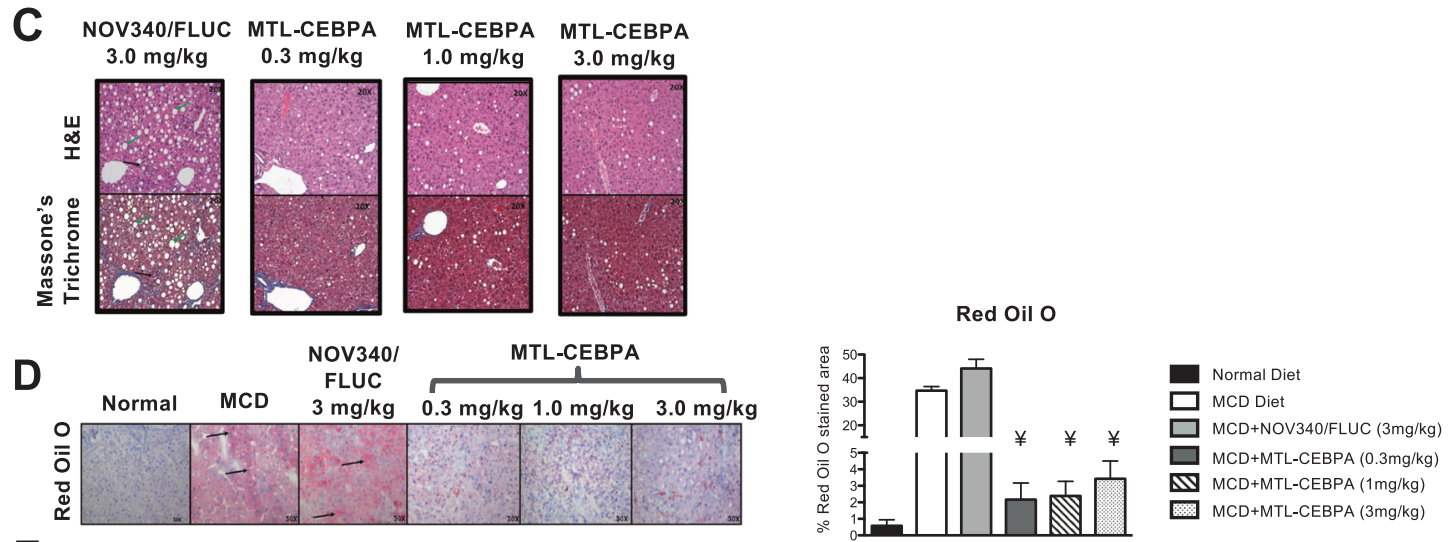

E
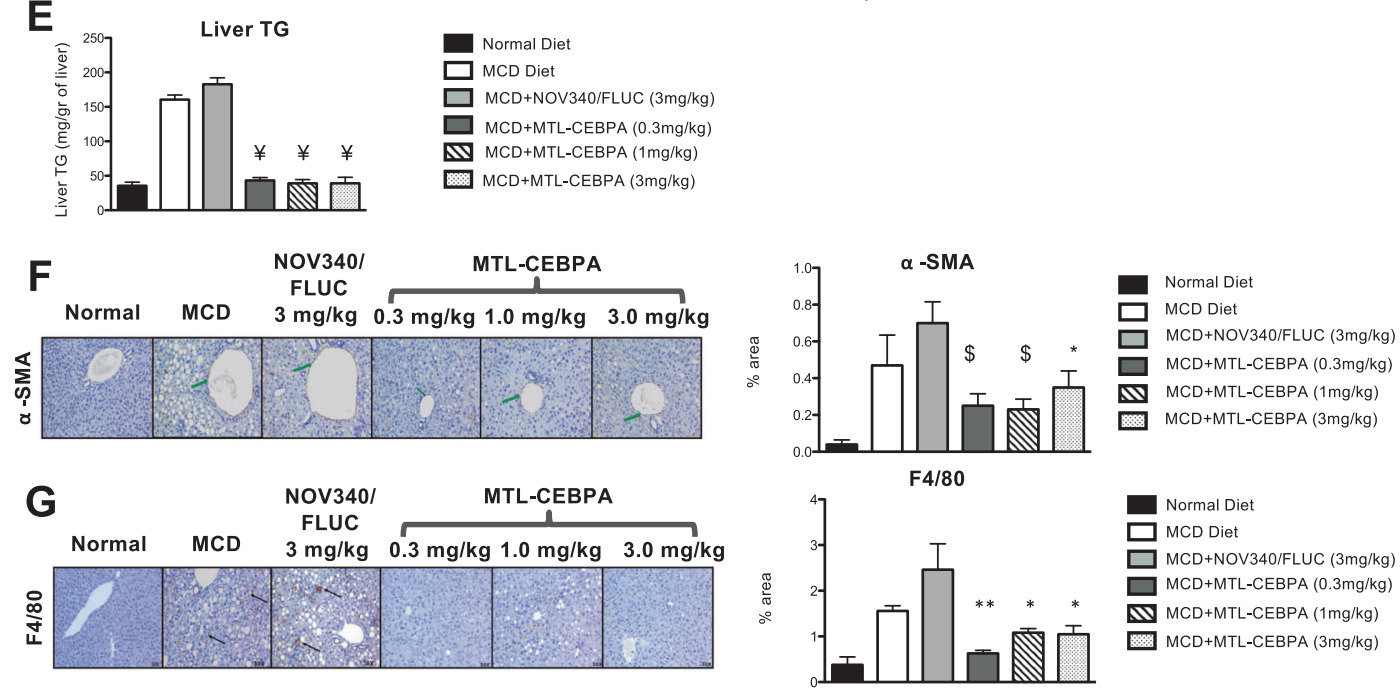

over-representation analysis of protein lysates from CEBPA-51-transfected HCC lines (HepG2; Hep3B, and PLCPRF5) were analyzed by mass spectroscopy; significant protein changes in pathways that regulated lipid metabolism were observed (Supplementary Fig. 1). We therefore investigated the short-term beneficial outcome of 
Fig. 5 The effects of MTL-CEBPA on MCD-induced fatty liver disease. a Histological analysis of liver sections from normal diet and MCD diet-fed animals for (a, upper panels) 4 weeks and (a, lower panels) 6 weeks stained with H\&E and Masson's trichome (magnification: $\times 20)$. (a, left panel) Analysis of liver sections from normal diet control animals showed normal architecture with hepatocytes arranged in the form of cords (blue arrow) radiating away from the central vein. The portal trip (red arrow) contained an artery, vein, and the bile duct. Normal distribution of the sinusoidal spaces (yellow arrow) was observed. (a, right panel) Analysis of the liver sections from MCD diet animals revealed steatosis (green arrow) and inflammatory cell infiltration (black arrow). b Serum levels of ALT and AST indicate reduction to near normal levels following 2 weeks of MTL-CEBPA treatment. Bilirubin levels do not continue to increase in the presence of 1 and $3 \mathrm{mg} / \mathrm{kg}$ of MTL-CEBPA treatment. $¥=p(0.0002) ; *=p$ (0.002); $\$=p(0.003)$. Histological analysis of liver sections from MCD diet-fed animals treated with NOV340/FLUC $(3 \mathrm{mg} / \mathrm{kg})$ and MTL-CEBPA at $0.3 \mathrm{mg} / \mathrm{kg} ; 1.0 \mathrm{mg} / \mathrm{kg}$ and $3 \mathrm{mg} / \mathrm{kg}$. c Liver sections stained with $H \& E$ and Masson's trichrome. (magnification: $\times 20$ ). Analysis of liver sections from MCD + NOV340/FLUC revealed steatosis (green arrow) and inflammatory cell infiltration (black arrow) which was more prominent, when compared with MCD diet at week 6 . The liver sections from all MTL-CEBPA-treated groups showed reduced steatosis and inflammatory changes, when compared to animals on MCD diet at week 6. The effect of MTL-CEBPA-51 on fat deposits in the liver. $\mathbf{d}$ The liver cryosections from animals administered with all doses of MTL-CEBPA were stained with Oil red O. Staining pattern (d, left panel) at magnification: $20 \times 20$ showed less staining intensity in all animals treated with MTL-CEBPA. The scoring of Oil red $\mathrm{O}$ staining as percentage of the section area stained (d, right panel) showed significant reduction in staining from the treated groups. $¥=p(<0.0002)$. e The effect of MTL-CEBPA treatment on liver triglyceride (TG) in MCD diet mice was also measured to consolidate the significant reduction seen from the Red Oil-O staining. Data are expressed as mean \pm SEM when compared to NOV340/FLUC. $¥=p(<0.0002)$. The effect of MTL-CEBPA on myofibrolast and macrophage formation. (f, left panel) Alpha SMA $(\alpha$-SMA) staining of liver sections comparing animals fed on normal and MCD diet at week 6 and the effects of MTL-CEBPA treatment. (magnification: $\times 20$ ). The liver sections from animals on normal diet revealed no sign of $\alpha$-SMA staining. Animals fed on MCD diet showed prominent staining (green arrow), which was significantly reduced in MTL-CEBPA treatment at all concentrations. Scoring of the area as a percentage value (f, right panel) showed significant reduction in staining from animals treated with MTL-CEBPA. (g, left panel) F4/80 staining analysis of liver sections comparing animals on normal vs. MCD diet at week 6 and the effects of MTL-CEBPA treatment at increasing doses (magnification: $\times 20$ ). The liver section revealed minimal F4/80 staining in the normal diet. More punctate staining was observed in the animals on MCD diet (black arrow). Scoring of the stained area (g, right panel) showed significant reduction in F4/80 at all doses of MTL-CEBPA groups compared to the control groups. Data are expressed as mean \pm SEM. $*=p(0.0026)$; $* *=p(0.0012) ; \$=p(0.0006)$

MTL-CEBPA in non-alcoholic fatty liver disease. Here the well-established methionine and choline-deficient diet (MCD) induced non-alcoholic steatohepatitis model was used. C57/BL6 mice were fed an MCD diet for 6 weeks to induce pathology. Liver sections confirmed distinct disruption to liver architecture with punctate steatotic lesions observed by week 4 (Fig. 5a). These represented accumulation of simple fat inclusions in the liver cells.
MTL-CEBPA treatment commenced at week 6 where NASH showed the most severe histopathological progression (Fig. 5a). MCD fed animals were divided into five groups ( $n=10$ in each) to follow increasing doses of MTLCEBPA treatment (bi-weekly for 2 weeks) at $0.3,1.0$, and $3.0 \mathrm{mg} / \mathrm{kg}$. This was compared with untreated MCD diet group and a NOV340/FLUC control treatment group. Treatment with MTL-CEBPA after 2 weeks caused a significant reduction in ALT and AST to near normal levels at all dose levels tested (Fig. 5b). Although bilirubin levels did not decrease significantly, they showed a trend towards reduction at higher doses of MTL-CEBPA treatment (Fig. $5 b)$. When we investigated H\&E- and Masson's trichromestained tissue sections of the liver, the MCD fed group treated with MTL-CEBPA at $0.3 \mathrm{mg} / \mathrm{kg} ; 1.0 \mathrm{mg} / \mathrm{kg}$, and $3.0 \mathrm{mg} / \mathrm{kg}$ showed major improvement in liver architecture at all concentrations (Fig. 5c). When the presence of lipid deposits was assessed by Oil red $\mathrm{O}$ staining, we observed a drastic reduction in positive staining only in the MTLCEBPA-treated groups to near normal levels when compared with the control group (Fig. 5d). This observation was also confirmed by quantifying liver triglyceride levels (Fig. 5e). This was similar to the $\mathrm{CCl}_{4}$-induced liver disease model (Fig. 3g) where we also observed improved clearance of fat deposits in MTL-CEBPA-treated animals.

Since liver damage typically triggers infiltration of macrophages and activation of myofibroblasts, we measured for the presence of both by staining FFPE-fixed liver tissue sections with anti- $\alpha$-smooth muscle actin and anti-F4/ F80 (Fig. 5f,g, respectively). Animals fed with MCD diet showed a significant increase in F4/80-positive macrophage staining, when compared with normal diet control. Treatment at all doses of MTL-CEBPA significantly decreased the percentage of positively stained macrophages.

\section{Discussion}

The findings presented here show development of a novel treatment for reversing liver fibrosis and liver steatosis through activation of CEBPA, a key liver transcription factor. Loss of CEBPA expression is a common feature in many forms of liver disease. This essential liver-enriched transcription factor is responsible for regulating the normal function and differentiation of hepatocytes and this is illustrated in many transgenic knock-out animal models that report development of severe liver injury; liver steatosis and hyperammonemia in the absence of CEBPA [9]. In a more clinically relevant setting, published reports demonstrate that suppressed CEBPA mRNA levels is followed by a reduction in CEBPA target gene expression in tumor vs adjacent normal tissues of HCC patients [16]. Lower levels of CEBPA mRNA, are correlated with shorter survival in 
HCC patients [17]. Paradoxically, there are also reports suggesting that higher CEBPA mRNA are observed in tumor vs adjacent normal tissue sections [18]. It must, however, be noted that C/EBP- $\alpha$ has two main isoforms (a p42 active and a p30 inactive form) which are posttranscriptionally regulated [19]. Therefore it is possible that these observed discrepancies are due to the detection of inactive forms of $\mathrm{C} / \mathrm{EBP}-\alpha$ and in this regard the downregulation of $\mathrm{C} / \mathrm{EBP}-\alpha$ target genes is a convincing reflection of the biological consequences in suppression of functional $\mathrm{C} / \mathrm{EBP}-\alpha$.

Gain of function studies, where CEBPA expression was exogenously enhanced, showed significant reduction in steatosis and reversal of liver damage [20]. Gene therapy approaches for increasing $\mathrm{C} / \mathrm{EBP}-\alpha$ levels using either hydronamic liver delivery or adenoviral systems also showed reduction in $\mathrm{CCl}_{4}$ induced liver fibrosis [7, 8]. CEBPA overexpression via adenovirus delivery showed elimination of orthotopic HCC xenograft nodules [11]. Similar observations have been made where CEBPA overexpression using recombinant adenoviral cassettes reduced tumourigenicity of HCC tumor cell lines [21]. Collectively, these published reports are consistent with the in vivo data we have presented here using synthetic saRNA oligonucleotides. Since other studies have also reported successful use of SMARTICLES to deliver oligonucleotides (microRNA mimetic and DNAi) to achieve antitumor activity both preclinically and clinically [14, 22], this further validates use of SMARTICLES as an attractive delivery vehicle. Liver activation of CEBPA through SMARTICLES (MTL-CEBPA) significantly improved liver function and enchanced survival in a $\mathrm{CCl}_{4}$-induced advanced liver cirrhotic model. MTL-CEBPA drastically reversed steatosis in a diet-induced NASH model and reduced tumor burden in a DEN-induced cirrhotic HCC model (consistent with our earlier publication where CEBPAsaRNA was delivered using PAMAM dendrimer nanoparticles [12]).

After treatment with MTL-CEBPA, we confirmed upregulation of CEBPA mRNA in the liver of both the DEN- and $\mathrm{CCl}_{4}$-treated models, which was also linked to upregulation of albumin; a downstream target gene of CEBPA. Upregulation of albumin by MTL-CEBPA confirms directly that elevation of CEBPA mRNA leads to a functional co-activation in at least one of its target genes. In this report, the control oligonucleotide sequence encapsulated in the SMARTICLES (NOV340/siFLUC (abbreviated to NOV340/FLUC)) showed no anti-fibrotic, anti-steatotic, or antitumor activities. NOV340/FLUC furthermore showed no improvement in liver function. This concludes that the liver function activity reported here was being driven by the specific CEBPA-saRNA sequence and also supports evidence of good hepatic delivery of MTL-
CEBPA using SMARTICLES. Although we have not yet examined the cellular distribution of MTL-CEBPA in these studies, our aim will be to fully define the liver cell populations impacted by the drug. Currently histological evidience shows that MTL-CEBPA has a significant impact on macrophage and stellate cell activity in diseased livers.

The mechanism of CEBPA-saRNA is still being unraveled. Currently, we have demonstrated saRNA strand specificity and reliance on Argonaute 2 (Ago2) [13]. We also demonstrated that it requires at least one component of the RNA-interacting activating complex [6]. The exciting preclinical data reported here led us to drive MTL-CEBPA into a detailed 1-month toxicology study in rats and cynomolgus monkeys which will be reported in detail in the future. The extended dosing in the $\mathrm{CCl}_{4}$ model and the maintenance of activity for several weeks post cessation of dosing suggest a less-frequent dosing schedule might be efficacious, but this will require additional studies in preclinical models. As far as we are aware, coventional gene therapy approaches to modulate C/EBP- $\alpha$ (with their inherent complexity and safety challenges) have not yet progressed to a clinical setting.

MTL-CEBPA has successfully progressed into a Phase 1 dose escalation study in patients with primary HCC and liver metastases from other cancers (ClinicalTrials. gov-NCT0271601).

We believe that the unique combination of effects MTLCEBPA has in enhancing liver function and preventing liver failure is suggestive of a potential breakthrough drug for chronic liver disease. We propose oligonucleotide therapy as a potentially novel and safe alternative to activate tumor suppressor genes.

\section{Materials and methods}

\section{Small activating RNA oligonucleotides were synthesized with the following sequence}

\section{CEBPA-51}

Sense strand: mGmCGmGUCAUUmGUCAmCUGGUC $\mathrm{mUmU}$;

Anti-sense strand: GACCAGUGACAAUGACCGC mUmU.

siFLUC: the control oligonucleotide siFLUC targets firefly luciferase and has no complementarity in mammalian genome.

Sense strand: mCmUmUAmCGmCmUGAGmUAmCm UmUmCGAdTpsdT;

Anti-sense strand: UCGAAGmUACUmCAGCGm UAAGdTpsdT. 
Oligonucletodies were synthesized by St Pharm at $99 \%$ HPLC purity. $m=2$ '-O-Methyl and $\mathrm{ps}=$ Phosphorothioate are the strand modifications to the duplex RNA. All sequences are shown are in the $5^{\prime}-3^{\prime}$ orientation.

\section{Cell line studies}

\section{AML12}

The AML12 (alpha mouse liver 12) cell line was established from hepatocytes from a mouse (CD1 strain, line MT42) transgenic for human TGF alpha and purchased from ATCC. Cells were cultured in a 1:1 mixture of Dulbecco's modified Eagle's medium and Ham's F12 medium with $0.005 \mathrm{mg} / \mathrm{ml}$ insulin, $0.005 \mathrm{mg} / \mathrm{ml}$ transferrin, $5 \mathrm{ng} / \mathrm{ml}$ selenium, $40 \mathrm{ng} / \mathrm{ml}$ dexamethasone, and $10 \%$ fetal bovine serum (FBS).

\section{Clone 9}

Clone 9 (K-9) is an epithelial cell line isolated in 1968 from normal liver taken from a young male rat and was purchased from ATCC. Cells were cultured in F-12K Medium supplemented with $10 \%$ FBS.

\section{HepG2}

HepG2 cells are immortalized human liver carcinoma cells derived from the liver tissue of a 15-year-old Caucasian male with a well-differentiated hepatocellular carcinoma. Cells were cultured in RPMI1640 medium supplemented with $10 \%$ FBS.

\section{Primary human hepatocytes}

Primary human hepatocytes (HMCPTS, Life technologies) were sourced from Donor ID: HU8200-A, a male AfricanAmerican 52-year-old. Same batch numbered cells were purchased frozen and thawed following the manufacturer's protocol:

Primary hepatocyte thawing medium: Cryopreserved Hepatocyte Recovery Medium was used for thawing each vial (Life Technologies, CM7000)

Primary hepatocyte-plating medium: Fetal bovine serum, heat inactivated $50 \mathrm{ml}$ (Life Technologies, 16140-071); insulin-transferrin-selenium $(100 \times)$-5ml (Life Technologies, 41400-045); HEPES (1 M)-5ml (Life Technologies,15630-056); L-Glutamine-Penicillin-Streptomycin solution-5ml (Sigma, G1146); Dexamethasone-40 ng/ml final concentration (Sigma, D8893); Phenol red-free William's E Medium (Life Technologies, A12176-01).
Primary hepatocyte maintenance medium: Primary hepatocyte maintenance supplement (Life Technologies, CM4000); human hepatocyte growth factor- $40 \mathrm{ng} / \mathrm{ml}$ final concentration (Sigma, H5791); epidermal growth factor$20 \mathrm{ng} / \mathrm{ml}$ final concentration (Sigma, E9644), Nicotinamide$2.5 \mu \mathrm{g} / \mathrm{ml}$ final concentration (Sigma N0636); Phenol redfree William's E Medium-500ml (Life Technologies, A12176-01).

\section{Transfection studies (established cell lines)}

For analyzing gene activation and protein expression, hepatocytes were seeded into 24-well plates at a density of $1 \times 10^{5}$ cells per well. Transfection was performed with lipofectamine 2000. CEBPA-saRNAs (CEBPA-51) or scrambled saRNAs (siFLUC), were added to the cells at a final concentration of $20 \mathrm{nM}$, following the manufacturer's instructions (Life technologies, Cat number 11668019). The treatment was repeated $24 \mathrm{~h}$ later and the cells were harvested at the 72-h time point.

\section{Transfection studies (primary human hepatocytes)}

Cells were transfected at $1 \times 10^{5}$ cells per well in a 24 -well collagen coated dish at a final oligonucleotide concentration of $50 \mathrm{nM}$ with $3 \mu \mathrm{l}$ of HiPerFect transfection reagent (Qiagen, 301704). The cells were incubated in plating media for $5 \mathrm{~h}$ to allow monolayer formation before replacing with maintenance media. For the second (forward) transfection, the same conditions were used as for reverse transfection. Maintenance media was used for the remaining duration of the experiment.

\section{RNA extraction and real-time PCR}

Total RNA was extracted for reverse transcription (QuantiFast Reverse transcription, Qiagen) and target cDNA amplification by real-time PCR (QuantiFast SYBR Green Master mix). The cDNA probes used we purchased as prevalidated QuantiTect SYBR Probes from Qiagen. Real-time PCR was performed with validated QuantiTect SYBR probe Qiagen or validated FAM probes from Applied Biosystems.

\section{Western blot}

Liver sections from three representative rats from the control and MTL-CEBPA-treated groups were processed for total protein extraction using Biosciences Tissue-PE-LB Buffer following manufacturer's instruction. A total of $10 \mu \mathrm{g}$ of protein were separated using SDS-PAGE. Samples were transferred onto PVDF membranes using a semi-dry transfer system (Biorad) for western blotting using anti-CEBPA (Abcam: ab40764) and Lamin B (Santa-cruz: sc6216). 


\section{Statistical analysis}

Statistical analysis was performed using one-way or two-way analysis of variance, followed by Dunnett's/ Tukey's multiple comparison test wherever applicable. Kruskal-Wallis test followed by Dunn's test was used for histological scores.

\section{The following in vivo studies are included in the supplement}

In vivo study 1: effects of administering the clinical candidate MTL-CEBPA in DEN-induced HCC.

In vivo study 2 and 3: effects of administering the clinical candidate MTL-CEBPA following induction of chronic liver failure by $\mathrm{CCl}_{4}$-comparing short-term MTL-CEBPA treatment and long-term MTL-CEBPA treatment.

In vivo study 4: the effects of administering the clinical candidate MTL-CEBPA following induction of NASH by methionine and choline-deficient diet.

\section{Haematoxylin-eosin staining procedure (Mayer's H\&E protocol)}

Liver sections were processed and stained with H\&E by following the standard procedures. Slides were deparaffinized and rehydrated by treatment through in series of $100 \%$ alcohol, $95 \%$ alcohol, and $70 \%$ alcohol. Slides were washed with distilled water. To improve stain quality, formalin fixed tissue slides were re- fixed in Bouin's solution for $1 \mathrm{~h}$ at $50^{\circ} \mathrm{C}$. Slides were rinsed with tap water for 5-10 min to remove the yellow coloration. Slides were stained with Weigert's iron hematoxylin working solution for $10 \mathrm{~min}$. After staining, slides were rinsed with tap water for 10 min and washed with distilled water. After washing, slides were then stained with Biebrich scarlet-acid fuchsin solution for $10-15 \mathrm{~min}$ and washed with distilled water. Slides were differentiated with phosphomolybdic-phosphotungstic acid solution for 10-15 min or until collagen was not red. Slides were then transferred directly (without rinsing) to aniline blue solution for staining for 5-10 min. The slides were then differentiated in $1 \%$ acetic acid solution for 2-5 min and washed with distilled water. Slides were quickly dehydrated through $95 \%$ ethyl alcohol, absolute ethyl alcohol, and cleared with xylene. Finally, slides were mounted with resinous mounting medium.

\section{Oil red 0 staining}

Oil red $\mathrm{O}$ is a fat-soluble dye that can be used to stain neutral triglycerides and lipids on frozen sections. Frozen livers were cryomatrix (thermo scientific) fixed.
Cryosections of $4-5 \mu \mathrm{m}$ in thickness were mounted on the glass slides and maintained at $-20^{\circ} \mathrm{C}$, until staining. These sections were air-dried and then fixed with formaldehyde for 5-10 min. Then slides were placed in absolute propylene glycol for 2-5 min and stained with $0.5 \%$ Oil red $\mathrm{O}$ stain. After staining, slides were rinsed with tap water for $10 \mathrm{~min}$ and washed with distilled water. The slides were then counterstained with Mayer's Hematoxylin for $1 \mathrm{~h}$, washed and mounted with glycerin. In this staining protocol, lipids/ fat globules are stained bright red, whereas nuclei are stained blue.

\section{F4/80 and $a-S M A$ immunohistochemistry}

Paraformaldehyde-fixed liver tissue sections were stained for F4/F80 (Thermo Scientific: PA5-MA1-91124 at 1:1000) and $\alpha$ smooth muscle actin (Thermo Scientific: PA5-19465 at 1:1000) to assess liver macrophages (Kupffer cells) and myofibroblast formation. Staining was performed following the standard procedure of antigen retrieval. Staining was quantified using Leica Qwin Software. From each slide $\times 20$ images were taken and the percentage area staining from each image was quantified from all the animals.

Acknowledgements This work was funded by MiNA Therapeutics.

Author contributions: V.R, K-W H, H.H, R.H., and D.B: designed the experiments. V.R, S.J, P.A, S.D, S.C., and J.V. performed the in vitro studies and analyzed the data. K-WH, V.L., and N.K. performed the DEN studies. V.R, S.J, S.D, P.A, P.J.M. assisted with in vitro work. P.S. designed the saRNA sequence. V.R, N.H, R.H, D. B: contributed to construction and writing of the manuscript. I.R, J.J.R assisted in manuscript editing. R.H, D.B, N.H managed execution of this study.

\section{Compliance with ethical standards}

Conflict of interest J.V., S.C., D.B., and R.H. are current or former employees of MiNA Therapeutics Limited. V.R., P.J.M., K.W.-H., P. S., J.J.R., and N.H. own stock in MiNA (Holdings) Limited. All remaining authors have no conflicts to declare.

Open Access This article is licensed under a Creative Commons Attribution-NonCommercial-NoDerivatives 4.0 International License, which permits any non-commercial use, sharing, distribution and reproduction in any medium or format, as long as you give appropriate credit to the original author(s) and the source, and provide a link to the Creative Commons license. You do not have permission under this license to share adapted material derived from this article or parts of it. The images or other third party material in this article are included in the article's Creative Commons license, unless indicated otherwise in a credit line to the material. If material is not included in the article's Creative Commons license and your intended use is not permitted by statutory regulation or exceeds the permitted use, you will need to obtain permission directly from the copyright holder. To view a copy of this license, visit http://creativecommons.org/licenses/by-nc-nd/4.0/. 


\section{References}

1. Ge PS, Runyon BA. Treatment of patients with cirrhosis. N Engl J Med. 2016;375:767-77.

2. Allen AM, Kim WR, Moriarty JP, Shah ND, Larson JJ, Kamath PS. Time trends in the health care burden and mortality of acute on chronic liver failure in the United States. Hepatology. 2016;64:2165-72.

3. Starley BQ, Calcagno CJ, Harrison SA. Nonalcoholic fatty liver disease and hepatocellular carcinoma: a weighty connection. Hepatology. 2010;51:1820-32.

4. Ekstedt M, Hagström H, Nasr P, Fredrikson M, Stål P, Kechagias $S$. et al. Fibrosis stage is the strongest predictor for diseasespecific mortality in NAFLD after up to 33 years of follow-up. Hepatology. 2015;61:1547-54.

5. Portnoy V, Huang V, Place RF, Li LC. Small RNA and transcriptional upregulation. WIREs RNA. 2011;2:748-60.

6. Portnoy V, Lin SHS, Li KH, Burlingame A, Hu Z-H, Li H, et al. saRNA-guided Ago2 targets the RITA complex to promoters to stimulate transcription. Cell Res. 2016;26:320-35.

7. Mei S, Wang X, Zhang J, Qian J, Ji J. In vivo transfection of C/ EBP-alpha gene could ameliorate CCL(4)-induced hepatic fibrosis in mice. Hepatol Res. 2007;37:531-9.

8. Tao L-L, Cheng Y-Y, Ding D, Mei S, Xu J-W, Yu J, et al. C/EBP$\alpha$ ameliorates $\mathrm{CCl}(4)$-induced liver fibrosis in mice through promoting apoptosis of hepatic stellate cells with little apoptotic effect on hepatocytes in vitro and in vivo. Apoptosis. 2012;17:492-502.

9. Hong I-H, Lewis K, Iakova P, Jin J, Sullivan E, Jawanmardi N, et al. Age-associated change of C/EBP family proteins causes severe liver injury and acceleration of liver proliferation after CCl4 treatments. J Biol Chem. 2014;289:1106-8.

10. Ee HT, Shing CH, Mirtha L, Esther W, Sathivel P, Aileen W. et al. CAAT/enhancer binding protein alpha knock-in mice exhibit early liver glycogen storage and reduced susceptibility to hepatocellular carcinoma. Cancer Res.2005;65:10330-7.

11. Shi Y-C, Zhao H, Yin C, Zeng X, Zhang Q, Xu W-P, et al. C/ EBP $\alpha$ inhibits hepatocellular carcinoma by reducing Notch3/ Hes1/p27 cascades. Dig Liver Dis. 2013;45:844-51.
12. Reebye V, Sætrom P, Mintz PJ, Huang K-W, Swiderski P, Peng L, et al. Novel RNA oligonucleotide improves liver function and inhibits liver carcinogenesis in vivo. Hepatology. 2014;59: 216-27.

13. Voutila J, Reebye V, Roberts TC, Protopapa P, Andrikakou P, Blakey DC et al. Development and mechanism of small activating RNA targeting CEBPA, a novel therapeutic in clinical trials for liver cancer. Mol Ther 2017;25:2705-14.

14. Rodrigueza WV, Woolliscroft MJ, Ebrahim A-S, Forgey R, McGovren PJ, Endert G, et al. Development and antitumor activity of a BCL-2 targeted single-stranded DNA oligonucleotide. Cancer Chemother Pharmacol. 2014;74:151-66.

15. Reebye V, Sætrom P, Mintz PJ, Huang K-W, Swiderski P, Peng L, et al. Novel RNA oligonucleotide improves liver function and inhibits liver carcinogenesis in vivo. Hepatology. 2014;59: 216-27.

16. Xu L, Hui L, Wang S, Gong J, Jin Y, Wang Y, et al. Expression profiling suggested a regulatory role of liver-enriched transcription factors in human hepatocellular carcinoma. Cancer Res. 2001;61:3176-81.

17. Tomizawa M, Watanabe K, Saisho H, Nakagawara A, Tagawa M. Down-regulated expression of the CCAAT/enhancer binding protein alpha and beta genes in human hepatocellular carcinoma: a possible prognostic marker. Anticancer Res. 2003;23:351-4.

18. Lu G-D, Leung CH-W, Yan B, Tan CM-Y, Low SY, Aung MO, et al. C/EBPalpha is up-regulated in a subset of hepatocellular carcinomas and plays a role in cell growth and proliferation. Gastroenterology. 2010;139:632-43.

19. Nerlov C. The C/EBP family of transcription factors: a paradigm for interaction between gene expression and proliferation control. Trends Cell Biol. 2007;17:318-24.

20. Inoue Y, Inoue J, Lambert G, Yim SH, Gonzalez FJ. Disruption of hepatic C/EBPalpha results in impaired glucose tolerance and agedependent hepatosteatosis. J Biol Chem. 2004;279:44740-8.

21. Watkins PJ, Condreay JP, Huber BE, Jacobs SJ, Adams DJ. Impaired proliferation and tumorigenicity induced by CCAAT/ enhancer-binding protein. Cancer Res. 1996;56:1063-7.

22. Bader AG. miR-34-a microRNA replacement therapy is headed to the clinic. Front Genet. 2012;3:120. 